\title{
GOÄ-Reform
}

\section{PKV will Verhandlungen aussitzen}

\author{
Das Zeitfenster für die GOÄ-Novelle schließt sich langsam, doch vor allem die Privatversicherer \\ spielen offenbar auf Zeit. Unter den Fachärzten macht sich langsam Nervosität breit.
}

$\longrightarrow$ ie Verhandlungen zur Novellierung der Gebührenordnung für Ärzte (GOÄ) kommen nicht voran, der PKV-Verband spielt auf Zeit. Die Fachärzte werden allmählich unruhig und befürchten, dass dieses Projekt in dieser Legislaturperiode noch scheitern könnte. Die Existenz so mancher Facharztpraxis steht und fällt mit den Umsätzen aus der Privatpraxis. Der Anteil der Privateinnahmen am Gesamtumsatz liegt im Durchschnitt aller Fachgruppen bei über 25\%, die Urologen verzeichnen einen Anteil von knapp $35 \%$ (siehe Grafik).

Es ist unverkennbar, dass der Verband der Privaten Krankenversicherung (PKV) und die Vertreter der Beihilfestellen kein ausgeprägtes Interesse daran haben, die GOÄ-Verhandlungen zu forcieren und zu einem erfolgreichen Abschluss zu führen. Die Verzögerungstaktik des PKVVerbandes ist insofern wenig plausibel, als die privaten Krankenkassen ein hohes Interesse daran haben sollten, das Geschäftsmodell einer umfassenden privaten Gesundheits-Vollversorgung zu stabilisieren und die niedergelassenen Ärzte dabei als Partner zu erhalten. Die

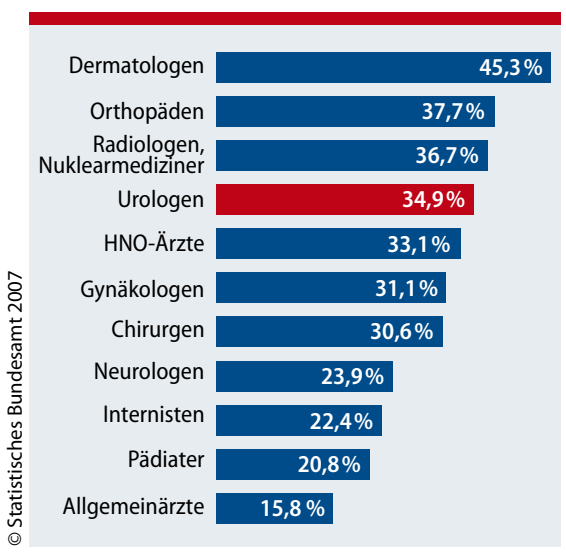

Grafik: Anteil der Einnahmen aus der Privatpraxis am Gesamtumsatz letzte Runde von Beitragserhöhungen hat den PKV-Unternehmen geschadet und die Zahl der „Nichtzahler“ im Versichertenbestand deutlich erhöht.

\section{Wissen alle, dass sie in einem Boot sitzen?}

Die Privatassekuranzen müssen damit rechnen, bei neuen politischen Konstellationen in Berlin in einer „Bürgerversicherung"von den gesetzlichen Krankenkassen „vereinnahmt“ zu werden. Die PKV-Unternehmen und die Ärzte befinden sich bei diesen Konstellationen gesundheitspolitisch in einem Boot. Diese gemeinsame Interessenlage ist bei der aktuellen Verhandlungsführung des PKVVerbandes noch nicht zu erkennen.

Die Forderungen der Ärzte zum Einstieg in die formellen GOÄ-Verhandlungen liegen seit Langem auf dem Tisch: —Aktualisierung des Leistungsverzeichnisses mit Anpassung an den medizinisch-technischen Fortschritt;

_ angemessene Vergütung der ärztlichen Grundleistungen (z.B. Beratungen, Untersuchungen, Besuche);

_Einbeziehung der „Analogen Bewertungen" in die GOÄ;

_ keine Übernahme der Vergütungsregelungen des EBM;

_ betriebswirtschaftliche Kalkulation der Bewertungen unter Berücksichtigung des ärztlichen Aufwandes und der Praxiskosten;

_ keine Öffnungsklausel für Einzelverträge der PKV-Unternehmen mit einzelnen Ärzten;

_Erhöhung des Punktwertes, gegebenenfalls mit einer Preisanpassungsklausel; — Aufrechterhaltung einer „Spannenregelung “ zur Berücksichtigung des individuellen diagnostischen und therapeutischen Aufwandes bei der Leistungserbringung;
— kontinuierliche Fortentwicklung des Leistungsverzeichnisses.

Gerade die niedergelassenen Fachärzte haben ein vitales Interesse daran, dass ihre Leistungen wenigstens bei der Behandlung der Privatpatienten angemessen vergütet werden.

\section{These der Kostenexplosion längst widerlegt}

Über die Anpassung der Vergütung und über die Neubeschreibung des ärztlichen Leistungsspektrums hinaus müssen auch die spezifischen Bedingungen der ambulanten Medizin abgebildet werden, fordert Dr. Wolfgang-Axel Dryden von der KV Westfalen-Lippe. „Die Kolleginnen und Kollegen bieten im Gegensatz zur Klinik nicht nur Facharztstandard, sondern die durchgängige persönliche Leistungserbringung durch einen Facharzt", so Dryden.

Der zentrale Streitpunkt bei der GÖ̈Novellierung ist die notwendige Anhebung des Punktwertes. Seit 1983 wurde der Punktwert nur um 14\% angehoben. Die Behauptung der PKV, es gebe bei den privatärztlichen Leistungen eine „Kostenexplosion“ ist längst widerlegt. Tatsächlich liegt der Anteil der ambulanten privatärztlichen Leistungen seit 15 Jahren durchschnittlich konstant bei etwa $24 \%$.

Die PKV-Unternehmen haben in den letzten Jahren davon profitiert, dass auf die notwendige Punktwertanpassung verzichtet wurde. Hier gibt es jetzt einen deutlichen Nachholbedarf. „Mit uns gibt es nur dann eine neue GOÄ, wenn der Zielkorridor beim Honorarplus zweistellig ist“, stellt Dr. Theodor Windhorst, Vorsitzender des GOÄ-Ausschusses der Bundesärztekammer klar. Norbert Fischer 